\title{
International standards to document remaining autonomic Function in persons with SCI and neurogenic bowel dysfunction: Illustrative cases
}

\author{
Lance L. Goetz $\mathbb{D}^{1} \cdot$ Anton $^{E^{2} m m a n u e l}{ }^{2} \cdot \operatorname{Klaus~Krogh}^{3}$ \\ Received: 8 September 2017 / Revised: 12 October 2017 / Accepted: 13 October 2017 \\ (C) International Spinal Cord Society 2018
}

\begin{abstract}
Introduction Neurogenic bowel dysfunction (NBD) is a highly prevalent problem after spinal cord injury, with potential for significant impact on health and quality of life. The international standards to document remaining autonomic function after SCI were developed to standardize communication between professionals regarding neurogenic bowel and other autonomic function after SCI. To improve understanding of the bowel subsection, illustrative cases are presented.

Case presentation Three cases are presented which illustrate differences in presentation and scoring of the elements in the data set based upon varying injury severity and location.

Discussion Determination of neurologic level of injury is insufficient for assessment of autonomic function and there is no direct method of assessment. Hence, surrogate makers are needed. The bowel subsection of the International standards to document remaining autonomic function in persons with SCI is an easy-to-use tool for this purpose.
\end{abstract}

\section{Introduction}

Neurogenic bowel dysfunction (NBD) is a major lifelimiting problem after spinal cord injury (SCI). NBD has also long been recognized as an area of low self-care competence following SCI $[1,2]$. Impairments in sensation of need for defecation, of volitional control, and the resulting potential for constipation and fecal incontinence cause distress, impact social function, and present management challenges [3].

In one large European study, the prevalence of fecal incontinence was stable at 18-19\% [4]. However, data show that up to $75 \%$ have episodes of incontinence at least once per year [5] and 95\% need some sort of assisted defecation

$\triangle$ Lance L. Goetz

Lancelgoetz@gmail.com

1 Spinal Cord Injury and Disorders Service, Hunter Holmes McGuire VA Medical Center and Department of Physical Medicine and Rehabilitation, Virginia Commonwealth University, Richmond, VA, USA

2 GI Physiology Unit, University College Hospital, London, UK

3 Neurogastroenterology Unit, Department of Hepatology and Gastroenterology, Aarhus University Hospital, Aarhus, Denmark
[3]. The potential also exists for under-reporting due to stigma. Constipation due to neurogenic bowel also has been seen to increase with aging after SCI [4].

The innervation of the gut is fundamentally different from other organs in the body. Most control of contractions and secretion is through reflex activity in the enteric nervous system located within the bowel wall. Parasympathetic activity enhances contractions and stimulates secretion, while sympathetic activity has the opposite effect. Parasympathetic innervation is primarily by the vagus nerve, which innervates the gut all the way to splenic flexure. Thus, the spinal cord, via the pelvic splanchnic nerves or "nervi erigentes" only provides parasympathetic innervation from the splenic flexure to the rectum, via sacral root levels S2-4. The external striated sphincter, which is normally under voluntary control, is supplied by the pudendal nerve via root levels S2-4, while the internal anal sphincter is under reflex control, mainly via the enteric nervous system. Although spinal cord innervation via the sacral outflow always has been described as parasympathetic, there is some animal evidence for a sympathetic origin [6]. Sensory fibers from the gut follow the parasympathetic and sympathetic nerves.

Sympathetic innervation to the gut originates in the thoracolumbar outflow of the spinal cord. Spinal cord 
sympathetic neurons form synapses at paravertebral, prevertebral, and other ganglia, whose neurons terminate in the gut. The overall effect of the sympathetic nervous system on gut motility is inhibitory, including a tonic inhibitory effect on mucosal secretion [7]. In persons with SCI, conditions or stressors resulting in increased sympathetic output (e.g., sepsis) are clinically seen to reduce gut motility.

The pathophysiology of NBD depends on the level of spinal cord injury. Subjects with supraconal lesions have increased tone of the distal colon and rectum while transit time is prolonged throughout the colorectum $[6,8,9]$. In contrast, subjects with conal or cauda equina lesions have a flaccid, hypotonic distal colon, and rectum with significantly prolonged transit of the distal colon and rectum $[6,8,9]$. Both groups have significantly reduced emptying of the rectosigmoid at defecation [10,11]. Data suggest that gastric emptying and small intestinal transit are also prolonged, and this may be related directly to the level of injury, or via distal transit delay causing upstream hold up of transit [12].

The international standards to document remaining autonomic function after SCI [13,14] were developed as a means to standardize communication between professionals regarding overall autonomic function. The bowel subsection has been demonstrated to have at least moderate inter-rater reliability [15]. There are three parameters in the autonomic data set which pertain to neurogenic bowel dysfunction. These are: (1) sensation of need for bowel movement, (2) ability to prevent stool leakage (continence), and (3) voluntary sphincter contraction. The elements are scored as follows: 2 for normal function, 1 for reduced or altered neurological function, and 0 for complete loss of control. If the examiner is unable to assess due to preexisting or concomitant problems, the client is rated at "NT" on a given parameter [13, 14].

While the international standards have been published and available for over 8 years [13], adoption of their use has been slow. To improve understanding of the benefits of these standards, we decided that a review of relevant cases would be beneficial. The cases presented below are intended to illustrate differences in presentation and scoring of the elements in the data set based upon varying injury severity and location.

\section{Case 1}

The client is a 29-year-old woman with C5 complete (AIS A) tetraplegia secondary to an automobile accident 3 years earlier. She is married and has two children. Her husband leaves the home for work during the day. She is dependent for bowel care, which is performed every evening by her husband or a paid caregiver. For her bowel care regimen, she uses initial removal of stool from the rectum, followed by a polyethylene glycol-based bisacodyl suppository and subsequent digital rectal stimulation every 5-10 min until no further results with two subsequent digital stimulation. She occasionally requires lidocaine jelly per rectum for mild autonomic dysreflexia.

She reports overall very good success with bowel care. She has occasional small amounts of mucus occurring after completion of bowel care but only rare incontinence associated with illness. She reports that she can often sense fullness and achiness in the left lower quadrant of her abdomen.

Physical examination reveals a slender woman in no apparent distress. Her abdomen is protuberant. Atrophy is evident in triceps, forearms compartment hands, trunk, pelvis, gluteal muscles, and lower extremities. Motor examination reveals $5 / 5$ elbow flexors, $2 / 5$ wrist extensors, and no motor function in elbow extensors, finger flexors, hand intrinsic muscles, or lower extremity muscles. Sensory examination reveals intact sensation through the C5 dermatome, decreased pin and light touch at the C6 dermatome, and no sensation to pin or light tough at $\mathrm{C} 7$ or below. There is no sensation in the perianal dermatomes to pin, touch, or pressure. Rectal exam likewise reveals absent light touch, pinprick, and deep anorectal pressure sensation. Voluntary anal sphincter contraction is absent. Rectal tone is increased with positive anal wink and bulbocavernosus reflex. A suprapubic catheter is present.

\section{Scoring per autonomic standards, bowel subsection}

For this client, a score of 1 on sensation of need for a bowel movement would be most accurate, since her sensation is reduced or altered. Sensation may be very subtle but it is always a subjective report by the patient. This case likely represents a patient with "discomplete" SCI [16], in which the person has a clinically complete (AIS A) injury but nevertheless presents with some sensation, in this case visceral. For stool leakage, the client would have a score of 0 for ability to prevent stool leakage (continence). Even if the client has perfectly managed neurogenic bowel with no accidents, the intent of the standards is to report the impact of the SCI on function, and not the clinical status of the patient. Even if social continence is excellent due to ideal management, the anticipated impact of the injury would be no physical function or ability to prevent stool leakage. Finally, this client would score 0 for voluntary sphincter contraction based on the physical examination.

\section{Case 2}

The client is a 26-year-old man with T6 AIS C injury due to a motor cycle accident occurring 6 years previously. He also incurred a mild traumatic brain injury at that time. He is 
unmarried, lives alone, and is independent with activities of daily living, bowel, and bladder care. He reports preserved pain sensation and sense of left colon fullness as well as the need to defecate. He also expresses a desire to be seen as "normal" and does not want to be on a scheduled bowel care regimen, including use of suppositories. He admits to frequent urge to have a bowel movement, sometimes with only gas, and has small bowel movements about twice daily after meals. He also admits incontinence occurring 2-3 times per month, especially after a large or fatty meal. He has moderate lower extremity spasticity, for which he takes baclofen $10 \mathrm{TID}$ and tizanidine $4 \mathrm{mg}$ BID and $8 \mathrm{mg}$ at bedtime.

On physical examination, sensation is present through the T6 dermatome, greatly reduced in T7-9, but recovers to intact light touch and sensation at the T10 dermatome through the perianal dermatomes. Manual muscle testing reveals hip flexors $1 / 5$, knee extensors $2 / 5$, ankle dorsiflexors 1 right and 0 left, long toe extensors absent bilaterally, and ankle plantarflexors $3 / 5$ bilaterally. On rectal examination, there is increased anal sphincter tone with resistance to finger insertion, weak (2/5) voluntary anal sphincter contraction, positive anal wink, and hyperactive BCR.

\section{Scoring per autonomic standards, bowel subsection}

For this client, a score of 2 on sensation of need for a bowel movement would be most accurate as pinprick and light touch sensation in the perianal area are preserved. For stool leakage, the client would have a score of 1 for ability to prevent stool leakage (continence), due to present but weak voluntary anal sphincter contraction. Finally, this client would score 1 for voluntary sphincter contraction based on the physical examination.

\section{Case 3}

The client is a 64-year-old woman who had acute surgery for a lumbar disc prolapse (L2/L3) 18 months ago. She can walk almost normally. Since surgery, she has no bladder control and depends on clean intermittent catheterization. She has no sensation of defection and reports episodes of fecal incontinence once or twice per week. Much of the time she has abdominal discomfort. Her bowel program includes digital evacuation of the rectum which she performs herself. Stimulant laxatives (Bisacodyl) is taken on demand, usually once per week. If more laxatives are taken, the frequency of fecal incontinence increases.

On physical examination, the perineal skin is normal. There is no perianal sensation of light touch, pinprick, or deep anal pressure and she is unable to feel digital examination. Tone in the anal canal is reduced and there is no voluntary contraction, anal wink, or bulbocavernosus reflex. The ampulla recti is full of moderately hard feces.

\section{Scoring per autonomic standards, bowel subsection}

Her score for "Sensation of need for a bowel movement" is 0 . The same are the scores for "Ability to prevent stool leakage" and "Voluntary sphincter contraction". Thus, the client has a complete conal/cauda equina lesion with respect to the bowel. The case illustrates how subjects with only minor motor dysfunction can have severe bowel dysfunction.

\section{Discussion}

The level of SCI is usually determined from assessment of somatic motor and sensory function using the ISNCSCI (International Standards for Neurological Classification of Spinal Cord Injury) standards [17]. This classification is insufficient for assessment of autonomic function. Hence, the international standards to document remaining autonomic function in persons with SCI were developed. With the exception of the external anal sphincter muscle and the mucosa of the distal anal canal, innervation of the gastrointestinal tract is entirely autonomic. The main components are the enteric, the sympathetic, and the parasympathetic nervous systems. Neurogenic bowel dysfunction has major consequences for quality of life and needs individual evaluation. The enteric nervous system is not directly affected by SCI. Likewise, parasympathetic innervation of most of the gastrointestinal tract is from the vagal nerve, and unaffected by SCI. The effects of sympathetic denervation of the bowel is less severe than parasympathetic. It is therefore likely that the segments of the gut most severely affected are the distal colon, the rectum and part of the anal canal.

Unfortunately, no reliable methods exist for direct assessment of autonomic innervation of the bowel. Anorectal manometry and the barostat may provide some evidence for either supraconal or conal/cauda equine lesions as well as the completeness of lesion [8, 18, 19]. However, variation between subjects is very large and there is substantial overlap between able bodied and subjects with SCI. Colorectal transit time can be determined by radiopaque markers. However, colorectal transit is not only affected by the neurological deficit but also by immobility, diet, medication, and time since lesion. Scintigraphic assessment of colorectal emptying at defecation has revealed major differences between able bodied and subjects with either supraconal [11] or conal/cauda equine lesions [10]. Unfortunately, the method is expensive and only for scientific use. 
Based on the considerations above, no objective methods qualify for easy and reliable assessment of bowel function after SCI. Hence, surrogate markers are needed. These should be easy to perform, reproducible, and easy to interpret. The working group behind the autonomic standards wanted the assessment to include both visceral sensory and motor function, and considered it important to include objective evaluation. Hence "Sensation of need for a bowel movement", "Ability to prevent stool leakage," and "Voluntary sphincter contraction" were chosen even though they reflect somatic motor and somatosensory function at least as much as autonomic function. Future development of valid and easy to use methods are needed to improve assessment of autonomic gastrointestinal function.

An accurate neurologic examination and consistent documentation of the elements discussed above, especially anal light touch and pin sensation vs. deep anal pressure, is critical to precisely describe an individual's impairments, in order to convey this information to subsequent examiners. Examiners who see individuals with spinal cord injury may come from a variety of medical specialties with varying training experiences, including neurology, physiatry, neurosurgery, urology, and medicine. Inconsistency exists in this examination and this is important to eliminate such inconsistencies [20].

The bowel section of the International standards to document remaining autonomic function in persons with SCI is very simple. If more detailed information is warranted, the Neurogenic Bowel Dysfunction score [21, 22] or the International SCI Bowel Function Data Set are recommended [22].

\section{Compliance with ethical standards}

Conflict of interest The authors declare that they have no competing interests.

\section{References}

1. Boss BJ, Pecanty L, McFarland SM, Sasser L. Self-care competence among persons with spinal cord injury. SCI Nurs Publ Am Assoc Spinal Cord Inj Nurses. 1995;12:48-53.

2. Stiens SA, Bergman SB, Goetz LL. Neurogenic bowel dysfunction after spinal cord injury: clinical evaluation and rehabilitative management. Arch Phys Med Rehabil. 1997;78(3 Suppl): S86-102.

3. Glickman S, Kamm MA. Bowel dysfunction in spinal-cord-injury patients. Lancet Lond Engl. 1996;347:1651-3.

4. Nielsen SD, Faaborg PM, Finnerup NB, Christensen P, Krogh K. Ageing with neurogenic bowel dysfunction. Spinal Cord. 2017;55:769-73.
5. Krogh K, Nielsen J, Djurhuus JC, Mosdal C, Sabroe S, Laurberg S. Colorectal function in patients with spinal cord lesions. Dis Colon Rectum. 1997;40:1233-9.

6. Denny-Brown D, Robertson E, An investigation of the nervous control of defecation. Brain. 1935;58(2):256-310.

7. Browning KN, Travagli RA. Central nervous system control of gastrointestinal motility and secretion and modulation of gastrointestinal functions. Compr Physiol. 2014;4:1339-68.

8. Krogh K, Mosdal C, Gregersen H, Laurberg S. Rectal wall properties in patients with acute and chronic spinal cord lesions. Dis Colon Rectum. 2002;45:641-9.

9. Krogh K, Mosdal C, Laurberg S. Gastrointestinal and segmental colonic transit times in patients with acute and chronic spinal cord lesions. Spinal Cord. 2000;38:615-21.

10. Krogh K, Olsen N, Christensen P, Madsen JL, Laurberg S, Colorectal transport during defecation in patients with lesions of the sacral spinal cord. Neurogastroenterol Motil J Eur Gastrointest Motil Soc. 2003;15(1):25-31.

11. Rasmussen MM, Krogh K, Clemmensen D, Bluhme H, Rawashdeh Y, Christensen P. Colorectal transport during defecation in subjects with supraconal spinal cord injury. Spinal Cord. 2013;51:683-7.

12. Fynne L, Worsøe J, Gregersen T, Schlageter V, Laurberg S, Krogh K, Gastric and small intestinal dysfunction in spinal cord injury patients. Acta Neurol Scand. 2012;125(2):123-8.

13. Alexander MS, Biering-Sorensen F, Bodner D, Brackett NL, Cardenas D, Charlifue S, et al. International standards to document remaining autonomic function after spinal cord injury. Spinal Cord. 2009;47:36-43.

14. Krassioukov A, Biering-Sørensen F, Donovan W, Kennelly M, Kirshblum S, Krogh K, et al. International standards to document remaining autonomic function after spinal cord injury. J Spinal Cord Med. 2012;35:201-10.

15. Davidson RA, Carlson M, Fallah N, Noonan VK, Elliott SL, Joseph $\mathrm{J}$, et al. Inter-rater reliability of the international standards to document remaining autonomic function after spinal cord injury. J Neurotrauma. 2017;34:552-8.

16. Finnerup NB, Gyldensted C, Fuglsang-Frederiksen A, Bach FW, Jensen TS. Sensory perception in complete spinal cord injury. Acta Neurol Scand. 2004;109:194-9.

17. Kirshblum SC, Burns SP, Biering-Sorensen F, Donovan W, Graves DE, Jha A, et al. International standards for neurological classification of spinal cord injury (revised 2011). J Spinal Cord Med. 2011;34(6):535-46.

18. Sun WM, MacDonagh R, Forster D, Thomas DG, Smallwood R, Read NW. Anorectal function in patients with complete spinal transection before and after sacral posterior rhizotomy. Gastroenterology. 1995;108:990-8.

19. MacDonagh R, Sun WM, Thomas DG, Smallwood R, Read NW. Anorectal function in patients with complete supraconal spinal cord lesions. Gut. 1992;33:1532-8.

20. Alexander M, Aslam H, Marino R. How do you do the International Standards for Neurological Control of SCI Anorectal Exam? Spinal Cord Ser Cases. 2017;3:17078. https://doi.org/10. 1038/s41394-017-0015-x.

21. Krogh K, Christensen P, Sabroe S, Laurberg S. Neurogenic bowel dysfunction score. Spinal Cord. 2006;44:625-31.

22. Krogh K, Emmanuel A, Perrouin-Verbe B, Korsten MA, Mulcahey MJ, Biering-Sørensen F. International spinal cord injury bowel function basic data set (Version 2.0). Spinal Cord. 2017;55:692-8. 